\title{
Die Bestimmung der Salpetersäure bei Milch, die durch Wasserstoffsuperoxyd konserviert ist.
}

\author{
Von \\ Dr. R. Strohecker.
}

Mitteilung aus dem Nahrungsmitteluntersuchungsamt (Vorsteher: Prof. Dr. J. Tillmans) des Städtischen Hygienischen Universitätsinstituts in Frank. furt a. M. (Direktor: Prof. Dr. M. Neifer.)

\section{[Eingegangen am 3. September 1917.]}

Nachdem durch die Anordnung der Reichsstelle für Speisefette vom 21. Dezember 1916 die Verwendung von Wasserstoffsuperoxyd zur Frischhaltung von Magermilch freigegeben worden war, ist nunmehr auch durch die Anordnung vom 1. Juni ds. Jrs. Wasserstoffsuperoxyd zur Frischhaltung der Vollmilch, soweit sie nicht zur Ernährung von Säuglingen in Betracht kommt, zugelassen. Diese beiden Bestimmungen sind insofern auch für die Milchkontrolle von Bedeutung, als bekanntlich Wasserstoffsuperoxyd mit Diphenylaminschwefelsäure eine intensive Blaufärbung liefert, somit also einen Gehalt an Nitraten bezw. Salpetersäure vortäuschen kann ${ }^{\mathbf{1}}$ ). Bei der durch die herrschende Futterknappheit bedingten vielfach völlig anormal zusammengesetzten Milch ist die Beeinflussung der Nitratprobe um so bedauerlicher, als gerade der Salpetersäuregehalt der Milch mitunter auszchlaggebend für die Feststellung einer Wässerung ist. Auf Veranlassung von Herrn Prof. Dr. Tillmans habe ich daher einige Versuche ausgeführt, um zu ermitteln, welche Mengen Salpetersäure das zugesetzte Wasserstoffsuperoxyd vortäuscht und auf welche. Weise der Nitratgehalt einer Milch trotz der Konservierung durch Wasserstoffsuperoxyd noch qualitativ und quantitativ bestimmt werden kann.

Die qualitative sowohl wie die quantitative Bestimmung der Salpetersüure in der Milch wurde in allen Fällen nach der von Tillmans und Splittgerber vorgeschlagenen Methode ${ }^{2}$ ) ausgeführt. Ein bestimmtes Volumen Milch wird danach mit der gleichen Menge Quecksilberchlorid-Salzsäurelösung (1 Teil 5\%-ige Quecksilberchloridlösung +1 Teil $2 \%$-iger Salzsäure) versetzt und umgeschüttelt. Das klare Filtrat dient zur quantitativen wie qualitativen Bestimmung der Salpetersäure. $1 \mathrm{ccm}$ des Serums bezw. $1 \mathrm{ccm}$ der für die colorimetrische Bestimmung hergestellten Verdünnungen wird mit $4 \mathrm{ccm}$ des von Tillmans vorgeschriebenen Diphenylreagenses gemischt $(0,85 \mathrm{~g}$ Diphenylamin $+190 \mathrm{ccm}$ verdünnte Schwefelsäure $1: 3$ werden mit salpetersäurefreier Schwefelsäure vom spez. Gew. 1,84 auf $500 \mathrm{ccm}$ aufgefüllt). Nach einer Stunde beobachtet man die entstandene Färbung.

Für den qualitativen Nachweis des Wasserstoffsuperoxydes in der Milch wurde bei den nachstehenden Versuchen die von Arnold und Mentzel ${ }^{3}$ ) empfohlene Titanschwefelsäure benutzt $(0,2 \%$ Titansäure in verdünnter Schwefelsäure). Die

1) Willeke, Schellenbach und Yilke, Diese Zeitschrift 1912, 24, 227-239. C. La ar, Ber. Deutsch. Chem. Gesellsch. 1882, 15, 2086. - Vergl. auch Tillmans, Diese Zeitschrift 1910, 20, 695.

2) Diese Zeitschrift 1911, 22, 401-405.

$\left.{ }^{3}\right)$ Diese Zeitschrift 1903, 6, 305. 
Reaktion kann in der Weise ausgeführt werden, daß man $10 \mathrm{cem}$ Mileh mit 10 bis 12 Tropfen des Reagenses versetzt. Bei Anwesenheit von 0,015-0,0175.g Wasserstoffsuperoxyd in $100 \mathrm{~cm}$ Milch soll eine deutliche Gelbfärbung wahrnehmbar sein. Empfindlicher ist nach unseren Versuchen die Reaktion, wenn man an Stelle der Milch das Quecksilberchlorid-Salzsäureserum verwendet ${ }^{1}$ ). Auf diese Weise machte sich noch ein Wasserstoffsuperoxydgehalt von $5 \mathrm{mg}$ im Liter durch eine schwache, aber deutlich wahrnehmbare Gelbfärbung des Serums bemerkbar. In destilliertem Wasser lief sich mit Hilfe des Titanreagenses noch $2 \mathrm{mg}$ Wasserstoffsuperoxyd im Liter nachweisen. Als Reagens auf Wasserstoffsuperoxyd ist die beschriebene Diphenylaminschwefelsäure empfindlicher; noch $1 \mathrm{mg}$ Wasserstoffsuperoxyd im Liter Milch lieferte eine schwache, aber noch deutlich erkennbare Blaufärbung. Da sich kleine Mengen Wasserstoffsuperoxyd in frischer Milch sehr schnell zersetzen, so wurde bei diesen Empfindlichkeitsprüfungen zunächst das Quecksilberchlorid-Salzsäureserum hergestellt und diesem erst kurz vor den Versuchen das Wasserstoffsuperoxyd zugesetzt.

Zunächst wurden folgende Vorversuche angestellt: 3 Milchproben (I, II und III) von gleicher Beschaffenheit, die weder gewässert noch mit Wasserstoffșuperoxyd konserviert waren, wurden nachträglich mit $50 \mathrm{ccm} 21 / 2 \%$-iger Wasserstoffsuperoxydlösung auf 1 Liter verseizt. Zu Probe I wurden außerdem nach der von dem hiesigen Untersuchungsamt angewandten Konservierungsmethode noch $8 \mathrm{ccm}$ einer $5 \%$-igen Quecksilberchloridlösung auf ein Liter $(=0,04 \%)$, zu Probe III eine bestimmte Menge einer Nitratlösung, entsprechend $16 \mathrm{mg}$ Salpetersäure $\left(\mathrm{N}_{2} \mathrm{O}_{5}\right)$ im Liter hinzugefügt. Kurz nach dem Zusatz der Wasserstoffsuperoxydlösung wurde von den drei Proben das Quecksilberchlorid-Salzsäureserum hergestellt und mittels des Diphenylaminreagenses geprüft. Die Diphenylaminschwefelsäure färbte sich bei allen Proben sofort tief blau, das Wasserstoffsuperoxyd täuschte somit einen hohen Gehalt an Salpetersäure vor. Nach der quantitativen Salpetersäurebestimmung würde die Blaufärbung einem Salpetersäuregehalt von über $200 \mathrm{mg}$ im Liter entsprochen haben. Nach 5 Stunden wurde das Serum nochmals hergestellt und geprüft. Bei allen drei Milchproben trat noch eine starke Blaufärbung ein. Nach 24 Stunden gab das Serum von Probe II nur noch eine schwache Blaufärbung, Probe I und III dagegen lieferten eine stark positive Diphenyl aminreaktion, die bei I $90 \mathrm{mg}$, bei II $19 \mathrm{mg}$ Salpetersäure im Liter entsprach. Mittels der Titanreaktion wurden in Probe II und III nur noch Spuren, in Probe I dagegen noch gröBere Mengen Wasserstoffsuperoxyd festgestellt. Diese Vorverauche zeigen zunächst, daß Wasserstoffsuperoxyd sehr große Mengen Salpetersäure nach der Diphenylaminreaktion vortäuschen $\mathrm{kann}$, femer, daß das Wasserstoffsuperoxyd sich rerhältnismäßig schnell in der gewöhnlichen, nicht anderweitig konservierten Milch zersetzt ${ }^{2}$ ).

In Probe III, der zu Anfang $16 \mathrm{mg}$ Salpetersäure im Liter hinzugefügt worden waren, konnte nach 24 Stunden, in welcher Zeit das Wasserstoffsuperoxyd bis auf Spureu zerstört wurde, der zugesetzte Salpetersäuregehalt annähernd (19 mg) wiedergefunden werden. Nach dieser letzten Beobachtung wäre theoretisch daher in einer mit Wasserstoffsuperoxyd konservierten Milch die qualitative bezw. quantitative Salpetersäurebe-

1) Vergl. auch Amberg, Journ. of Biol. Chem. 1906, 1, 219; diese Zeitschrift 1907, 13,571 .

2) H. Chick, Zentralbl. Bakteriol. II. Abt. 1901, 705-717; diese Zeitschrift 1902, 5, 420. - E. Feder. Diese Zeitschrift 1908, 15, 234-236. - E. Baumann, Münch. med. Wochenschr. 1908, 1083. - Hinks, Analyst, 1915, 40, 482 und Pharm. Zentralhalle 1916, 57, $803-804$. 
stimmung mit Hilfe der Diphenylreaktion möglich, wenn man nur die Milch solange sich selbst überließe, bis das zugesetzte Wasserstoffsuperoxyd zersetzt ist. Dem steht jedoch erstens entgegen, daß sich geringe Mengen Wasserstoffsuperoxyd bei anfäng. lich großem Zusatz des Reagenses sehr lange unzersetzt in der Milch erhalten, auch dann, wenn die Milch schon säuert ${ }^{1}$ ), was eintritt, wenn die Hauptmenge des Wasserstoffsuperoxyds zerstört ist. Wichtiger als dieser Einwand ist jedoch, daß der Salpetersäuregehalt in geronnener oder saurer Milch sehr schnell völlig oder wenigstens größtenteils verschwindet ${ }^{2}$ ). Um den Salpetersäuregehalt nit der Diphenylaminreaktion nachzuweisen, bleibt daher nur der andere Weg übrig, nämlich, das Wasserstoffsuperoxyd durch chemische Umsetzung zu beseitigen. Ich stellte auch einige Versuche an, um zu sehen, ob durch Kochen der Wasserstoffsuperoxydgehalt der Milch entfernt werden könnte. Dies ist jedoch nicht der Fall. Der Wasserstoffsuperoxydgehalt wurde durch Kochen der Milch nur wenig verringert, geschweige denn zerstört.

Als praktisch erwies sich die Zersetzung des Wasserstoffsuperoxyds durch 1/10 N.-Kaliumpermanganatlösung, zumal sich damit gleichzeitig eine quantitative Bestimmung des in der Mileh enthaltenen Wasserstoffsuperoxyds verbinden ließ.

In schwefelsaurer bezw. salpetersaurer Lösung wirken Permanganat und Wasserstoffsuperoxyd bekanntlich nach folgender Gleichung aufeinander ein:

$2 \mathrm{KMnO}_{4}+5 \mathrm{H}_{2} \mathrm{O}_{2}+4 \mathrm{H}_{2} \mathrm{SO}_{4}=2 \mathrm{KHSO}_{4}+2 \mathrm{MnSO}_{4}+8 \mathrm{H}_{2} \mathrm{O}+5 \mathrm{O}_{2}$.

Aus den weiter unten angeführten Versuchen ist ersichtlich, daß die bei der Umsetzung entstehenden Produkte auf die Diphenylaminreaktion ohne Einfluß sind. Verwendet man zur Zerstörung des Wasserstoffsuperoxyds eine 1/10-N.-Permanganatlösung, wie sie in der Maßanalyse zur Bestimmung des Wasserstoffsuperoxydgehaltes angewendet wird, so entspricht $1 \mathrm{em} 1 / 10$ N.Permanganatlösung $0,0017 \mathrm{~g}$ Wasserstoffsuperoxyd. Unzerstörtes Kaliumpermanganat bewirkt an sich wie wohl jedes Oxydationsmittel eine Blaufärbung der Diphenylaminschwefelsäure. Ein Überschuß von Permanganat muß daher vermieden werden. Die völlige Zerstörung des Wasserstoffsuperoxyds wird dadurch angezeigt, daß beim Zutropfen des Permanganats die Rosafärbung nicht mehr verschwindet. Der hierfür notwendige Überschuß an Permanganat ist äuberst gering und verschwindet nach kurzer Zeit infolge der Oxydation des noch vorhandenen Milchzuckers, sodaß für die nachfolgende Diphenylreaktion der Permanganatüberschuß ohne Bedeutung ist.

Die Versuche wurden in folgender Weise angestellt:

$\mathrm{Zu}$ einer Reihe von Milchproben, I, II, III, IV, V und VI, von denen I und IV mit $0,04 \%$ Sublimat konserviert, III mit $20 \mathrm{mg}$ Salpetersäure im Liter versetzt war, wurden je 50 ccm einer 2,52\% sodaB die Proben zu Anfang $1260 \mathrm{mg}$ Wasserstoffsuperoxyd im Liter enthielten ${ }^{3}$ ). Probe VI wurde vor dem Wasserstoffsuperoxydzusatz aufgekocht. $20 \mathrm{ccm}$ (entsprechend $10 \mathrm{ccm}$ Milch) der in der üblichen Weise gewonnénen Quecksilberchlorid-Salzsäureseren

1) H. Chick, Zentralbl. Bakteriol. II. Abt. 1901, 705-717; diese Zeitschrift 1902, 5, 420. - E. Feder, diese Zeitschrift 1908, 15, 234-236. - E. Baumann, Münch. med. Wochenschr. 1903, 1083. - Hinks, Analyst 1915, 40, 482 und Pharm. Zentralhahe 1916, 57, $803-804$.

2) Vergl. auch Tillmans u. Schneehagen. - Diese Zeitschrift 1916, 31, 343-351.

3) Um Vollmilch für die Dauer von 24 Stunden haltbar zu machen, sieht die Anordnung der Reichsfettstelle vom 1. Juni 1917 einen Zusatz von $33-50 \mathrm{ccm}$ einer $3 \%$ o-igen Wasserstoffsuperoxydlösung auf ein Liter Mileh vor. 
wurden mit $10 \mathrm{ccm}$ verdünnter Schwefelsäure $(1+3)$ versetzt und mit $1 / 10$ N.Permanganatlösung titriert, bis die Rosafärbung kurze Zeit bestehen blieb. Die verbrauchte Anzahl ccm Permanganatiösung mit 170 multipliziert, ergibt die in einem Liter Milch vorhandene Menge Waszerstoffsuperoxyd in $\mathrm{mg}$. Ein nachträgliches Verschwinden der Rosafärbung bleibt unberücksichtigt, da es, wie oben erwähnt, auf der Oxydation des Milchzuckers beruht. Das auf diese Weise von Wasserstoffsuperoxyd befreile Serum wurde nunmehr der qualitativen oder quantitativen Diphenylaminreaktion unterworfen. Für die quantitative Bestimmung empfiehlt es sich der Einfachheit halber, dem titrierlen Serum aus einer Bürette noch soviel destilliertes Wasser hinzuzufügen, daß das Volumen von $40 \mathrm{~cm}$ erreicht wird, da ja die Verdünnungen berücksichtigt werden müssen. (20 ccm Serum $+10 \mathrm{ccm}$ Schwefelsäure + a ccm 1/10 N.-Permanganatlösung $+(10-$ a) $\mathrm{ccm}$ dest. Wasser). Die Versuchsergebnisse sind in den Tabellen Nr. I und II zusammengestellt:

Tabelle $I$.

(Die Versuche sind bei Zimmertemperatur ausgeführt.)

\begin{tabular}{|c|c|c|c|c|c|c|c|c|c|}
\hline $\begin{array}{l}\text { Bezeichnung } \\
\text { der Proben }\end{array}$ & Nach Stunden & $1 / 4$ & 2 & 4 & 24 & 48 & 72 & 120 & 168 \\
\hline \multirow{4}{*}{$\begin{array}{c}\text { Probe I } \\
\text { Zusatz: } 0,04 \% \\
\text { Quecksilber- } \\
\text { chlorid + } 1260 \\
\text { mg/ Wasser- } \\
\text { stofsuperoxyd }\end{array}$} & $\begin{array}{c}\text { Wasserstoff- } \\
\text { superoxyd } \mathrm{mg} / \mathrm{l}\end{array}$ & 1190 & 1072 & 1004 & 944 & 884 & 867 & 867 & 867 \\
\hline & Säuregrad & $6,6^{\circ}$ & - & - & $6,8^{\circ}$ & $6, \delta^{\circ}$ & $6,8^{\circ}$ & - & - \\
\hline & $\begin{array}{c}\text { Alkohol- } \\
\left.\text { probe }^{1}\right)\end{array}\left\{\begin{array}{l}a \\
b\end{array}\right.$ & - & $\left\{\begin{array}{l}\text { n. ko- } \\
\text { aguliert } \\
\text { koagu- } \\
\text { liert }\end{array}\right.$ & - & $\left\{\begin{array}{c}\text { n. ko- } \\
\text { aguliert } \\
\text { koagu- } \\
\text { liert }\end{array}\right.$ & - & - & - & - \\
\hline & $\begin{array}{l}\text { Salpetersäure } \\
\left(\mathrm{N}_{2} \mathrm{O}_{5}\right) \mathrm{mg} / \mathrm{l}\end{array}$ & 0 & 0 & 0 & 0 & 0 & 0 & 一 & - \\
\hline \multirow{4}{*}{$\begin{array}{c}\text { Probe If } \\
\text { Zusatz: } 1260 \mathrm{mg} \\
\text { Wasserstoff- } \\
\text { superoxyd }\end{array}$} & \begin{tabular}{|c|} 
Wasserstoff- \\
superoxyd $\mathrm{mg} / \mathrm{l}$
\end{tabular} & 968 & 492 & 246 & 43 & \multicolumn{4}{|c|}{ Titanreaktion negativ } \\
\hline & Sìuregrad & $6,0^{\circ}$ & - & - & $6,0^{\circ}$ & $9,0^{\circ}$ & \multicolumn{3}{|c|}{ geronnen } \\
\hline & $\begin{array}{c}\text { Alkohol- } \\
\left.\text { probe }{ }^{1}\right)\end{array}\left\{\begin{array}{l}a \\
b\end{array}\right.$ & - & $\begin{array}{c}\text { nieht } \\
\text { koagu- } \\
\text { liert }\end{array}$ & - & $\left|\begin{array}{c}\text { nieht } \\
\text { kougu- } \\
\text { liert }\end{array}\right|$ & $\begin{array}{c}\text { nieht } \\
\text { koagu- } \\
\text { liert }\end{array}$ & - & - & - \\
\hline & $\begin{array}{l}\text { Salpetersäure } \\
\left(\mathrm{N}_{2} \mathrm{O}_{5}\right) \text { mg/l }\end{array}$ & 0 & 0 & 0 & 0 & 0 & 0 & - & - \\
\hline \multirow{4}{*}{$\begin{array}{c}\text { Probe III } \\
\text { Zusatz: } 1260 \mathrm{mg} / \mathrm{l} \\
\text { Wasserstoff- } \\
\text { superoxyd }+20 \\
\text { mg// Salpeter- } \\
\text { säure in Form } \\
\text { von Nitrat }\end{array}$} & \begin{tabular}{|c|}
\multicolumn{2}{|c|}{ Wasserstoff- } \\
superoxyd $\quad \mathrm{mg} / \mathrm{l}$
\end{tabular} & 766 & 442 & 156 & 15 & \multicolumn{4}{|c|}{ Titanreaktion negativ } \\
\hline & Sänregrad & $5,2^{0}$ & - & - & $5,4^{0}$ & $\begin{array}{l}30,4 \text { (ye- } \\
\text { rommen) }\end{array}$ & \multicolumn{3}{|c|}{ geronnen } \\
\hline & $\begin{array}{c}\text { Alkohol- } \\
\left.\text { probe }^{1}\right)\end{array}\left\{\begin{array}{l}a \\
b\end{array}\right.$ & - & $\begin{array}{c}\text { nicht } \\
\text { koagu- } \\
\text { liert }\end{array}$ & - & $\begin{array}{l}\text { nicht } \\
\text { koagu- } \\
\text { liert }\end{array}$ & - & - & - & - \\
\hline & $\begin{array}{l}\text { Salpetersäure } \\
\left(\mathrm{N}_{2} \mathrm{O}_{5}\right) \mathrm{mg} / \mathrm{l}\end{array}$ & 21 & 18 & 22 & 20 & 0 & 0 & - & - \\
\hline
\end{tabular}

1) a bedeutet die Alkoholprobe mit dem einfachen, b mit dem doppelten Volumen $70 \%$ igen Alkohols. 
Tabelle Il.

(Die Versuche sind bei Zimmertemperatur ausgeführt.)

\begin{tabular}{|c|c|c|c|c|c|c|c|c|}
\hline $\begin{array}{l}\text { Bezeichnung } \\
\text { der Proben }\end{array}$ & Nach Stunden & $1 / 4$ & $1 / 2$ & 1 & 2 & 24 & 48 & 96 \\
\hline \multirow{3}{*}{$\begin{array}{c}\text { Probe IV } \\
\text { Zusatz: } 0,04 \% \\
\text { Quecksilber- } \\
\text { chlorid + } 1245 \\
\text { mgll Wasser- } \\
\text { stoffsuperoxyd }\end{array}$} & $\begin{array}{c}\text { Wasserstoff- } \\
\text { superoxyd mgl } 1\end{array}$ & 1165 & - & 一 & 918 & 909 & 833 & 799 \\
\hline & Säuregrad & $5,8^{\circ}$ & $5,8^{0}$ & $5,8^{\circ}$ & 5,80 & $5,8^{\circ}$ & - & $6,8^{\circ}$ \\
\hline & Alkoholprobe $\left\{\begin{array}{l}\mathrm{a} \\
\mathrm{b}\end{array}\right.$ & $\begin{array}{l}\text { D. Koaguliert } \\
\text { koaguliert }\end{array}$ & - & - & $\begin{array}{c}\text { n.konguliert } \\
\text { koaguliert }\end{array}$ & - & - & - \\
\hline \multirow{3}{*}{$\begin{array}{c}\text { Probe V } \\
\text { Zusatz: } 1245 \mathrm{mg} / 1 \\
\text { Wasserstoff- } \\
\text { superoxyd }\end{array}$} & \begin{tabular}{|c|} 
Wasserstoff- \\
superoxyd $\mathrm{mg} / 1$
\end{tabular} & 867 & - & - & 417 & 68 & \multicolumn{2}{|c|}{$\begin{array}{c}\text { Titanreaktion } \\
\text { negativ }\end{array}$} \\
\hline & Säuregrad & $5,6^{\circ}$ & $5,2^{\circ}$ & $48^{\circ}$ & $4,8^{0}$ & $5,0^{3}$ & \multicolumn{2}{|c|}{ geronnen } \\
\hline & Alkoholprobe $\left\{\begin{array}{l}\mathrm{a} \\
\mathrm{b}\end{array}\right.$ & $\left|\int \begin{array}{c}\text { nicht } \\
\text { kaaguliert }\end{array}\right|$ & - & - & $\left\{\begin{array}{c}\text { niclit } \\
\text { koaguliert }\end{array}\right.$ & $\begin{array}{c}\text { nicht } \\
\text { koagnliert }\end{array}$ & - & - \\
\hline \multirow{3}{*}{$\begin{array}{c}\text { Probe VI } \\
\text { Zusatz: } 1245 \mathrm{mg} / 1 \\
\text { Wasserstoff- } \\
\text { superoxyd, vor- } \\
\text { her aufgekocht }\end{array}$} & $\begin{array}{c}\text { Wasserstoff- } \\
\text { superoxyd } \mathrm{mg} / \mathrm{l}\end{array}$ & 1222 & - & - & 1122 & 1083 & 1020 & 986 \\
\hline & Säuregrad & $4,9^{\circ}$ & $4,9^{\circ}$ & $4,9^{\circ}$ & $5,0^{\circ}$ & $5,0^{0}$ & - & $5,4^{0}$ \\
\hline & Alkoholprube $\left\{\begin{array}{l}\mathrm{a} \\
\mathrm{b}\end{array}\right.$ & - & - & - & $\begin{array}{l}- \\
-\end{array}$ & - & - & $\begin{array}{l}- \\
-\end{array}$ \\
\hline \multirow{3}{*}{$\begin{array}{c}\text { Probe } 0 \\
\text { Kein Zusatz }\end{array}$} & $\begin{array}{c}\text { Wasserstoff- } \\
\text { superoxyd } \mathrm{mg} / \mathrm{l}\end{array}$ & 0 & 0 & 0 & 0 & 0 & 0 & 0 \\
\hline & Säuregrad & $5,2^{0}$ & $5,6^{0}$ & $5,8^{\circ}$ & - & \multicolumn{3}{|c|}{ geronnen } \\
\hline & Alkoholprobe $\left\{\begin{array}{l}a \\
b\end{array}\right.$ & $\left\{\begin{array}{c}\text { nicht } \\
\text { koaguliert }\end{array}\right.$ & - & - & $\left\{\begin{array}{c}\text { nicht } \\
\text { koaguliert }\end{array}\right.$ & - & - & - \\
\hline
\end{tabular}

Die Versuche zeigen zunäehst, daP sich Wasserstoffsuperoxyd in der mit Quecksilberchlorid versetzten Milch sehr lange unzersetzt erhält; nach 7 Tagen konnten in dem einen Falle mit Hilfe der Permanganattitration noch etwa $69 \%$ der zugefügten Menge nachgewiesen werden. Die ausschließlich durch Wasserstoffsuperoxyd konservierten Proben enthielten nach 24 Stunden nur noch sehr geringe Mengen_ unzerstörtes Wasserstoffsuperoxyd (etwa 1-4\% der zugesetzten Menge). Nach $48^{-}$Stunden fiel die Titanreaktion in diesen Proben negativ aus. Da jedoch, wie oben angedeutet, nach der beschriebenen Methode nur noch $5 \mathrm{mg}$ Wasserstoffsuperoxyd im Liter nachgewiesen werden können, so ist es immerhin möglich, daß sich in den Milchproben trotz des negativen Ausfalls der Reaktion Spuren von unzersetztem Reagens erhalten hatten, was nit den Beobachtungen von Chick, Feder und anderen Forsehern übereinstimmen würde, zumal in diesen Fällen die Diphenylaminreaktion ohne vorherige Behandlung des Serums mit Permanganat meist noch schwach positiv ausfiel. Weiter bestätigen die Versuche, daß die Diphenylaminreaktion durch die zur Zerstörung des Wasserstoffsuperoxyds zugesetzten Reagenzien bezw. durch die bei der Umsetzung sich bildenden Verbindungen nicht gestört wurde. In den Fällen I und II wurden nach der Bestimmung mit Permanganat stets negative Reaktionen erhalten. Probe III, der einige mg Nitrat zugefügt worden waren, lieferte dagegen nach der Zerstörung 
des Wasserstoffsuperoxyds durch Permanganat eine starke positive Reaktion, und zwar entsprach die Blaufärbung, nach der colorimetrischen Methode bestimmt, 18 bis $22 \mathrm{mg}$ Salpetersäure im Liter; $20 \mathrm{mg}$ auf das Liter waren der Mileh zugesetzt worden. Die Übereinstimmung genügt vollkommen den Anforderungen. Von Probe III wurde nach 24 Stunden das Quecksilberchlorid-Salzeäureserum ohne vorherige Entfernung des noch vorhandenen Wasserstoffsuperoxyds der quantitativen Salpetersäurebestimmung unterworfen, die erbaltene Blaufärbung entsprach $18 \mathrm{mg}$ Salpetersäure im Liter. Aus diesem Versuch geht hervor, daß die geringen Mengen Wasserstoffsuperoxyd, die noch in der Milch vorhanden sind, die quantitative Salpetersäurebestimmung nicht oder nur wenig beeinflussen. Probe II, die nach 24 Stunden noch $43 \mathrm{mg}$ Wasserstoffsuperoxyd enthielt, lieferte ohne Beseitigung des Reagenses noch eine positive Diphenylaminreaktion. Probe III zeigte nach 48 Stunden einen sehr hohen Säuregrad und war geronnen; wie anzunehmen, war der Salpetersäuregehalt in dieser gesäuerten Milch völlig verschwunden. Aus dieser Beobachtung ergibt sich, daß in mit Wasserstoffsuperoxyd versetzter Mileh der Nachweis von Salpetersäure mit Sicherheit nur geführt werden kann, so lange noch genügende Mengen des Konservierungsmittels zugegen sind, um die Säuerung zu unterbinden. Hieraus erhellt ein neuer Vorteil des Quecksilberchlorids als Konservierungsmittel bei der Milchkontrolle. Abgesehen davon, daß der Quecksilberchloridzusatz den Säuregrad konstant bält und dadurch noch nach mehreren Tagen eine Salpetersäurebestimmung ermöglicht ${ }^{1}$ ), kann in der bei der Probenentnahme mit Quecksilberchlorid konservierten Milch der Wasserstoffsuperoxydgebalt noch nach längerer Zeit bestimmt und damit die anfänglich zugesetzte Menge beurteilt werden.

Tabelle II zeigt außerdem, daß sich Wasserstoffsuperoxyd in gekiochter Milch nur äußerst langsam zersetzt, was ja erklärlich ist, da in solcher Milch sowobl Enzyme wie auch Bakterien zerstört bezw. abgetötet sind. Diese Feststellung steht im Gegensatz zu den Beobachtungen von $\mathrm{Amberg}{ }^{2}$ ), der angibt, daß auch gekochte Milch Wasserstoffsuperoxyd rasch zersetzt. Vielleicht erklärt sich diese Differenz daraus, $\mathrm{da} a$ bei den von A mberg angestellten Versuchen schnell wieder Keime in die abgekochte Milch gelangt sind, die eine rasche Zerlegung des Wasserstoffsuperoxydes bewirkten; bei meinen Versuchen wurde die Milch nach dem Abkochen in einer verschlossenen Flasche aufbewahrt.

Ein merkwürdiges Verhalten des Säuregrades wurde bei Probe $\mathrm{V}$ beobachtet. Während die ursprüngliche Milch eiñen Säuregrad von 5,20 aufwies, zeigte die mit Wasserstoffs neroxyd versetzte Milch zunächst $5,6^{0}$, nach $1 / 2$ Stunde $5,2^{\circ}$ und nach einer Stunde $4,8^{\circ}$; erst nach 24 Stunden hatte der Säuregrad wieder eine kleine Steigerung erfahren. Der gegenüber der ursprünglichen Milch erböhte Säuregrad in Probe V von 5, $6^{\circ}$ zu Anfang der Beobachtung ist wohl darauf zurückzuführen, daß der verwendeten Wasserstoffsuperoxydlösung eine geringe Menge Säure zugefügt worden war, um sie besser haltbar zu machen. Das Fallen des Säuregrades in den darauf folgenden Stunden kann nur so erklärt werden, daß durch den Wasserstoffsuperoxydzusatz die Bildung alkalisch reagierender Körper begünstigt wird, die dann erst nach $2 \notin$ Stunden von der anfänglich gehemmten Wirkung der Milchsäurebakterien überholt wird.

1) Vergl. Tillmans, Splittgerber und Riffart. - Diese Zeitschrift 1914, 27, 893 bis 901 .

2) Journ. of Biol. Chem. 1906, 1, 219-228; diese Zeitschrift 1907, 13, 571. 
Merkwürdig erscheint ferner die Beobachtung, daß die mit Quecksilberchlorid und Wasserstoffsuperoxyd konservierten Milchproben wohl die einfache, jedoch nicht die doppelte Alkoholprobe aushielten, während die ursprüngliche Milch sowohl wie die mit Wasserstoffsuperoxyd und die mit Quecksilberchlorid allein versetzten Proben bei der einfachen und der doppelten Alkoholprobe längere Zeit nicht koagulierten.

\title{
Zusammenfassung der Ergebnisse.
}

1. Wie schon früher festgestellt wurde, täuscht Wasserstoffsuperoxyd in der Milch bei der Ausführung der Diphenylaminschwefelsäurereaktion grobe Mengen Salpetersäure vor. In Gegenwart von Wasserstoffsuperoxyd kann daher die Reaktion nicht ohne weiteres ausgeführt werden.

2. Die qualitative und quantitative Salpetersäurebestimmung kann mittels der Diphenylaminschwefelsäurereaktion in der bisher üblichen Weise vorgenommen werden, wenn man das vorhandene, mit Hilf̧e der Titanreaktion nachweisbare Wasserstoffsuperoxyd durch eine 1/10 N..Permanganatlösung unter Zusatz von verdünnter Schwefelsäure zerstört.

3. Mit der Salpetersäurebestimmung läßt sich ohne weiteres die Bestimmung des Wasserstoffsuperoxyds verbinden, indem man eine gemessene Menge des wach Tillmans und Splittgerber gewonnenen Quecksilberchlorid-Salzsäureserums mit Schwefelsäure ansäuert und mittels $1 / 10$ N.-Kaliumpermanganatlösung bis zur bleibenden Rosafärbung titriert. $1 \mathrm{ccm} 1 / 10 \mathrm{~N}$.-Kaliumpermanganatlösung entspricht $0,0017 \mathrm{~g}$ Wasserstoffsuperoxyd.

4. In dem nach Tillmans und Splittgerber bereiteten QuecksilberchloridSalzsäureserum lassen sich mit Hilfe dss Titanreagenses $(0,2 \mathrm{~g}$ Titansäure in verdünnter Schwefelsäure) noch $5 \mathrm{mg}$, mit Hilfe des Diphenylaminreagenses noch $1 \mathrm{mg}$ Wasserstoffsuperoxyd im Liter Milch nachweisen.

5. Es wurde ein merkwürdiges Verhalten des Säuregrades der mit Wasserstoffsuperoxyd konservierten Milch beobachtet.

\section{Die maßanalytische Bestimmung des Calciums im Trink- und Gebranchswasser.}

\author{
Von \\ Dr. J. Groffeld in Recklinghausen. \\ [Eingegangen am 8. September 1917.]
}

Die Bestimmung des Calciums im Wasser, die für seine Beurteilung hinsichtlich der Brauchbarkeit zu Trink- und Gebrauchszwecken von größter Bedeutung ist, erfolgt bekanntlich am sichersten als Oxalat auf gewichtsanalytischem oder maßanalytischem Wege. Die maßanalytischen Verfahren übertreffen die erstgenannten erheblich an rascher Ausführbarkeit und werden in der Praxis aus diesem Grunde gern vorgezogen.

Die bisher für den genannten $Z$ weck vorgeschlagenen Arbeitsvorschriften leiden alle an einer mohr oder minder großen Umständlichkeit, Sie zu vereinfachen, ohne ihre Genauigkeit auch in der Hand des weniger erfahrenen Analytikers zu beein- 